\title{
Precision time and the rotation of the Earth
}

\author{
Dennis D. McCarthy \\ U. S. Naval Observatory, Washington, DC 20392, USA \\ email: dmc@maia.usno.navy.mil
}

\begin{abstract}
Practical measurement of the passage of time requires the notion of a repeating phenomenon. The Earth's rotation has traditionally fulfilled this requirement. To cope with the impracticality of making precise measures of the Sun's hour angle or altitude, particularly in uncooperative weather conditions, various devices have been employed, but all have been calibrated with respect to astronomical phenomena related to the Earth's rotation, or to its orbital motion with respect to the Sun. Modern requirements for timing precision coupled with an increased understanding of the variability of the Earth's rotational speed are likely to bring about a change in the traditional relationship between precise timekeeping and astronomy. The historical background of this relationship and the current definitions are reviewed to show the development of timekeeping capabilities and the growing need for precise timekeeping. Possible future developments are outlined along with their advantages and disadvantages.
\end{abstract}

\section{Introduction}

Two components are involved in our measurement of time. First, we make use of a phenomenon perceived to be repeatable with sufficient regularity to serve our purpose. Second, we need to devise a convenient means to label the repetitions of this phenomenon. These elements correspond to the two properties of time measurement: interval and epoch. Historically we have used the repetitive cycles of celestial phenomena to serve as the basis for our time. The rising and setting of the Sun has provided the day; the phases of the Moon have provided the month and the position of the Sun with respect to the stars gives us the year.

These processes met historical timekeeping requirements with the exception of the times when the skies were not visible. For those times, various forms of clocks were created, and these were calibrated in terms of the traditional astronomical phenomena. When the need for extremely precise time arose, it became apparent that the Earth's rotation was not sufficient as the repeatable phenomenon. Even still we do maintain a relationship between clocks and astronomy. The background and issues involved are presented in detail in Nelson et al. (2001). This paper presents a summary of that paper and further explores the possibilities for the future.

\section{Historical background}

Time reckoned by the Earth's rotation has fulfilled the needs of society for timekeeping throughout history. The details of both astronomical time and "clock" time as implemented since the middle of the twentieth century are important in understanding the current timekeeping issues.

\subsection{Astronomical time}

Two primary methods of measuring time astronomically have been in common use for modern applications. The first is Universal Time, the time scale based on the rotation 
of the Earth on its axis. The second is characterized by a set of Dynamical Time Scales based on the revolution of the Earth in its orbit around the Sun.

\subsubsection{Universal time}

The form of Universal Time designated UT1 is the measure of astronomical time defined by the rotation of the Earth on its axis with respect to the Sun. It is nominally equivalent to mean solar time on the Greenwich meridian and reckoned from midnight. The mean solar day is traditionally described as the time interval between successive transits of the fictitious mean Sun over a given meridian. Historically, the unit of time, the mean solar second, was defined as 1/86 400 of a mean solar day (McCarthy 1991). In practice, Universal Time is determined, not by the meridian transit of the mean Sun, but by the observation of astronomical objects in conjunction with a conventional formula specifying UT1 in terms of Greenwich Mean Sidereal Time (GMST). The defining relation for UT1 with respect to the traditional optical reference system is given in Aoki et al. (1982). Beginning in January, 2003, a new defining expression has been implemented (Capitaine et al. 2000, 2003).

Apparent solar time, as determined by using a sundial or from measuring the altitude of the Sun, is the local time defined by the diurnal motion of the actual Sun. However, because the Earth's axis is not perpendicular to its orbital plane and because of its varying distance from the Sun, the time interval between successive meridian passages is not constant. This difference between mean and apparent solar time, called the equation of time, varies with the maximum being about $16 \mathrm{~min}$. Until the early nineteenth century, apparent solar time was used as the argument for astronomical ephemerides. However, as clocks improved, apparent solar time was gradually replaced by mean solar time. Variations in the Earth's rate of rotation were clearly established in the 1930s when a seasonal variation was observed in comparisons of astronomical observations with accurate pendulum and quartz crystal clocks (Scheibe \& Adelsberger 1936; Stoyko 1937). The time scale denoted UT2 was developed by adopting a conventional expression for the seasonal variation in the Earth's rotation, and this time scale was used briefly as a more uniform time scale determined from the Earth's rotation.

The sidereal day, which represents the Earth's period of rotation with respect to the stars is approximately 86164.0905 mean solar seconds. Due to precession the sidereal day is about $0.0084 \mathrm{~s}$ shorter than the actual period of rotation in inertial space. Thus, the true rotational period of the Earth is approximately 86164.0989 mean solar seconds. However, the mean solar day presently exceeds a day of exactly 86400 SI (The International System of Units (SI) 1998) seconds by about a millisecond. Therefore, the Earth's period of rotation is currently about 86164.0999 SI seconds.

The principal technique used to determine UT1 now is Very Long Baseline Interferometry (VLBI) observations of quasars. Although satellite laser ranging and tracking of GPS satellites provide estimates of UT1, because of the motion of satellite orbital nodes in space, VLBI provides the only rigorous determination of UT1.

\subsubsection{Dynamical time scales}

Ephemeris Time (ET) is a theoretically uniform time scale defined by the Newtonian laws of motion. When it became apparent that time based strictly on the Earth's rotation did not provide a uniform time scale, Clemence (1948) recommended a new standard of time based on the period of revolution of the Earth around the Sun, as represented by Newcomb's Tables of the Sun published in 1895. The measure of astronomical time defined in this way was given the name Ephemeris Time (ET). The working definition of Ephemeris Time was through Newcomb's formula for the geometric mean longitude 
of the Sun for the epoch of January 0, 1900, 12h UT (Newcomb 1895a),

$$
L=2794148.04+129602768.13 T+1.089 T^{2},
$$

where $\mathrm{T}$ is the time in Julian centuries of 36525 days. The proposal was adopted by the International Astronomical Union in 1952 (Trans. Int. Astron. Union, Vol. VIII 1954). Using the value of the linear coefficient in Newcomb's formula, the tropical year of 1900 contains 31556925.9747 s, so the International Committee for Weights and Measures (CIPM) in 1956 defined the second of Ephemeris Time to be "the fraction 1/31556925.9747 of the tropical year for 1900 January 0 at 12 hours ephemeris time."

Even though it was defined in terms of the longitude of the Sun, ET was determined in practice by using conventional lunar ephemerides in comparing observations of the positions of the Moon with respect to stellar backgrounds. Thus, secondary time scales, denoted ET0, ET1, and ET2, were defined by the ephemeris actually used in making the estimation of ET (Guinot 1989).

The benefit of a more uniform time scale provided by ET, however, was complicated by the fact that it was not easily accessible. That fact, combined with the development of atomic time scales, meant that Ephemeris Time never became a significant practical time scale. However, ET did replace UT1 as the independent variable used in astronomical ephemerides beginning in 1960 .

The concept of time scales based on the dynamics of the solar system was refined in 1976 when the International Astronomical Union (IAU) defined time-like arguments consistent with the general theory of relativity (Trans. Int. Astron. Union, Vol. XVIB 1977). This led to the development of Terrestrial Dynamical Time (TDT) and Barycentric Dynamical Time (TDB) (Trans. Int. Astron. Union, Vol. XVIIB 1980) that distinguish coordinate systems with origins at the center of the Earth and the center of the solar system, respectively.

In 1984 TDT replaced ET as the tabular argument of the fundamental geocentric ephemerides. It has an origin of 1 January $19770 \mathrm{~h}$ TAI (International Atomic Time), with a unit interval equal to the SI second, and maintains continuity with ET. In 1991 the IAU renamed TDT Terrestrial Time (TT). A practical realization of TT in terms of the atomic time scale, TAI, is (Explanatory Supplement to the Astronomical Almanac, rev. ed. 1992)

$$
T T=T A I+32.184 \mathrm{~s} .
$$

The constant offset represents the difference between ET and UT1 at the defining epoch of TAI on 1 January 1958.

TDB was defined to be used as the time-like argument for ephemerides referred to the barycenter of the solar system. By adopting an appropriately chosen scaling factor, TDB varies from TT or TDT by only periodic variations, with amplitudes less than $0.002 \mathrm{~s}$.

In 1991 the 21st IAU General Assembly introduced the general theory of relativity explicitly as the theoretical basis for the celestial reference frame and the form of the space-time metric to post-Newtonian order was specified. (Trans. Int. Astron. Union, Vol. $X X I B$ 1992). At that time it also clarified the definition of Terrestrial Time and further refined the concept of dynamical time by defining two new time scales, Geocentric Coordinate Time (TCG) and Barycentric Coordinate Time (TCB) (Seidelmann \& Fukushima 1992). The "coordinate" time scales TCG and TCB are complementary to the "dynamical" time scales TT (or TDT) and TDB. They differ in rate from TT and are related by four-dimensional space-time coordinate transformations (IERS Conventions 1996). These definitions were further clarified by resolutions adopted at the 24th IAU General Assembly in 2000 (Trans. Int. Astron. Union, Vol. XXIVB). The dynamical 


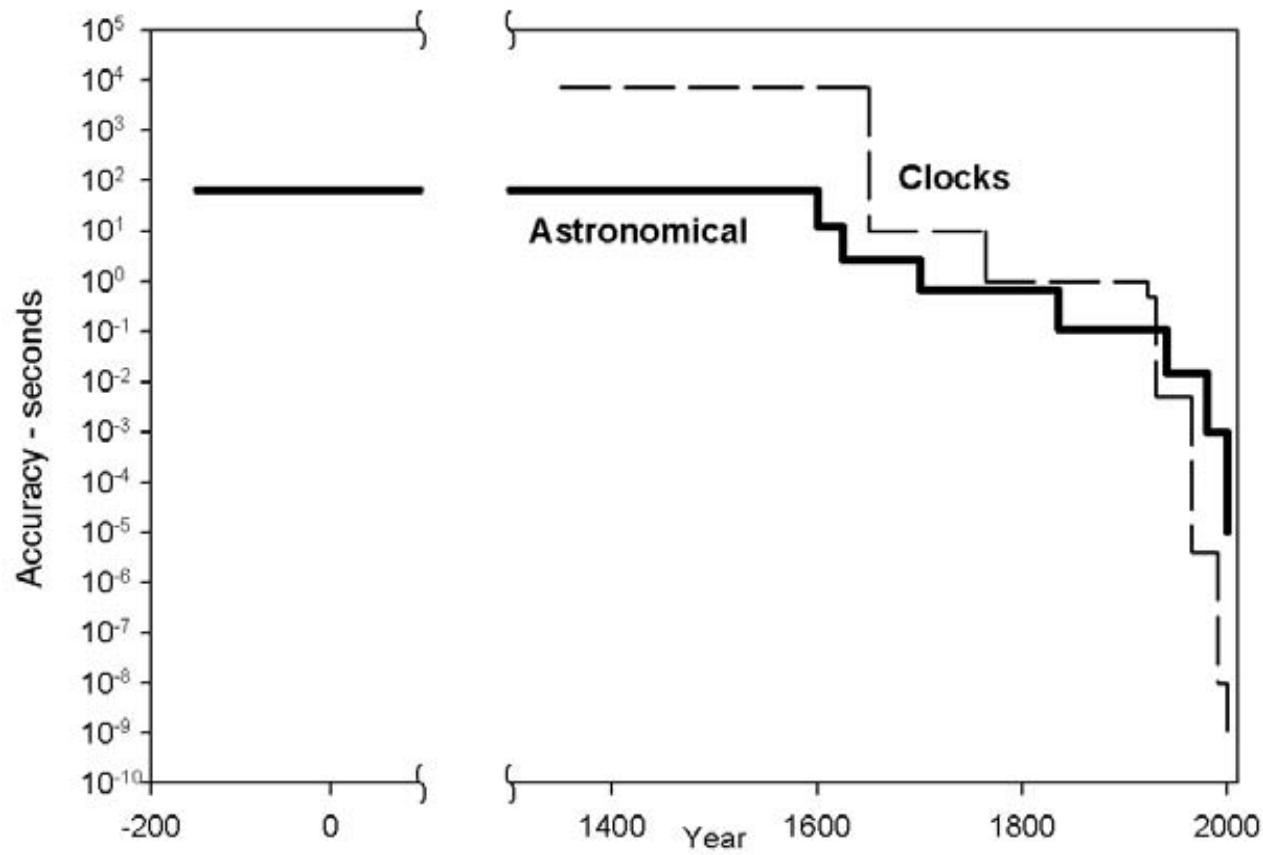

Figure 1. Development of timekeeping accuracy.

time scales are now used only for specialized studies and to develop astronomical ephemerides.

\section{2. "Non-astronomical" time}

To provide the means to measure the passage of time when it was not possible to use the Sun or stars, we have made use of various devices including clepsydrae, candles, and various forms of clocks. In the era of telescopic observations, pendulum clocks served as the standard means of keeping time until the introduction of modern electronics. Quartz crystal clocks were developed in the 1920s and 1930s (Gerber \& Sykes 1966), and the first atomic clock was constructed in 1948 (Natl. Bur. Stand. (U.S.) Tech. News Bull. 1949) using the microwave absorption line of ammonia to stabilize a quartz oscillator. In 1955 Louis Essen and J. V. L. Parry (Essen \& Parry 1955) constructed a practical caesium beam atomic clock and commercial caesium frequency standards appeared a year later. Norman Ramsey developed the hydrogen maser at Harvard University in 1960 (Goldenberg et al. 1960). Following the advent of atomic clocks, national timekeeping laboratories began to establish time scales based solely on atomic time, and these were coordinated by the Bureau International de l'Heure (BIH) (Guinot 2000). Some form of atomic time has been maintained continuously since 1955 (Guinot 1994/1995). The Bureau International des Poids et Mesures (BIPM) now has the responsibility for the maintenance of the standard international atomic time scale. Fig. 1 shows the development of the accuracy of astronomical and clock time scales with time. 


\subsubsection{Atomic time}

Following the appearance of the first operational caesium beam frequency standard in 1955 at the National Physical Laboratory (NPL) in the United Kingdom (Essen \& Parry 1957), the Royal Greenwich Observatory (RGO), U.S. Naval Observatory (USNO), and U. S. National Bureau of Standards (NBS) began to produce atomic time scales. The epochs of the USNO and NBS scales were made coincident and set equal to the observed UT2 on 1 January 1958 (Barnes et al. 1965). The details of the development of these scales into the current standard TAI (International Atomic Time) are contained in (Nelson et al. 2001).

\subsubsection{Atomic definition of the second}

Louis Essen and J. V. L. Parry of the NPL, in cooperation with William Markowitz and R. G. Hall at the USNO, determined the frequency of the NPL caesium standard with respect to the second of Ephemeris Time. Photographs of the Moon and surrounding stars were taken using the USNO dual-rate Moon camera over the period 1955.50 to 1958.25 to determine the Ephemeris Time from the position of the Moon at a known UT2. This information was used to calibrate the caesium beam atomic clock at NPL via simultaneous observations of the intervals between time pulses broadcast by radio stations WWV (then in Greenbelt, Md.) and GBR (Rugby, UK). The measured caesium frequency was $9192631770 \mathrm{~Hz}$ with a probable error of $20 \mathrm{~Hz}$ (Markowitz et al. 1958).

In October 1967 the atomic second was adopted as the fundamental unit of time in the International System of Units. It was defined as (Metrologia 1968) "the duration of 9192631770 periods of the radiation corresponding to the transition between the two hyperfine levels of the ground state of the caesium 133 atom," thus making the second of atomic time equivalent to the second of Ephemeris Time in principle.

\subsubsection{Establishment of TAI}

The Comité Consultatif pour la Définition de la Seconde (CCDS) of the CIPM recommended guidelines for the establishment of International Atomic Time in 1970. It stated that "International Atomic Time (TAI) is the time reference coordinate established by the Bureau International de l'Heure on the basis of readings of atomic clocks operating in various establishments in accordance with the definition of the second, the unit of time of the International System of Units" (Metrologia 1971). The CCDS (BIPM Com. Cons. Déf. Seconde 1970) defined the origin so that TAI would be in approximate agreement with UT2 on 1 January 1958, 0 h UT2.

This definition was refined in 1980 to account for relativistic concerns with the statement, "TAI is a coordinate time scale defined in a geocentric reference frame with the SI second as realized on the rotating geoid as the scale unit" (Metrologia 1981). TAI, when formally adopted in 1971, was an extension of the BIH atomic time scale that had been continuous back to 1955. In 1988, responsibility for maintaining TAI was transferred from the BIH to the BIPM. Today approximately two hundred clocks maintained in fifty laboratories contribute to the formation of TAI.

\section{Variations in the earth's rotational speed}

Astronomical observations have shown that the Earth's rotation does not provide a uniform time scale. The secular deceleration as well as a wide spectrum of quasi-random and periodic fluctuations have been well documented (Lambeck 1980). The low-frequency variations in the Earth's rotation are demonstrated by the difference in time, $\Delta \mathrm{T}$, between the uniform scale of Ephemeris Time or Terrestrial Time and the variable scale 


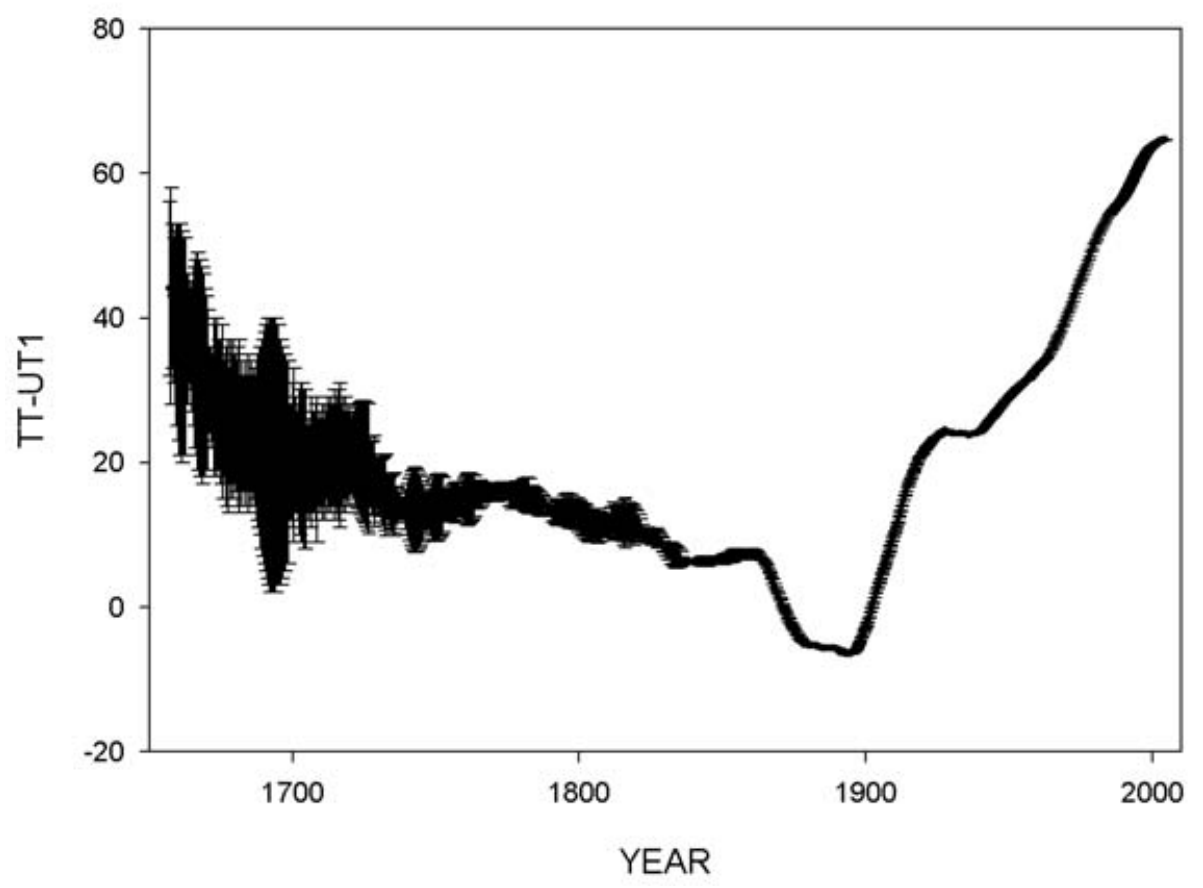

Figure 2. Variation of T since 1620 (in seconds).

of Universal Time. Values of $\Delta \mathrm{T}$ have been summarized (Morrison \& Stephenson 1986; McCarthy \& Babcock 1986). Before 1955, the values are provided by observations based on observations of the Moon. After 1955, they are determined using atomic clocks. Fig. 2 shows the observations since 1620 .

Stephenson (1997) shows that $\Delta \mathrm{T}$ over the past 2700 years can be represented approximately by a parabola of the form

$$
\Delta T=\left(31 s / c y^{2}\right)(T-1820)^{2} /(100)^{2}-20 s,
$$

where $\Delta \mathrm{T}$ is expressed in seconds and $\mathrm{T}$ is the year. The derivative of $\Delta \mathrm{T}$,

$$
L O D=(0.0017 s / d / c y)(T-1820) / 100,
$$

represents the excess length of day (the difference between the length of the astronomical day and $86400 \mathrm{SI}$ seconds) corresponding to the expression for $\Delta \mathrm{T}$ above. According to this long-term trend, the rate of increase in the length of the day is about $1.7 \mathrm{~ms}$ per century. Fig. 3 illustrates observations of changes in the length of day during the era of telescopic observations, from 1620 onwards. Over this period, LOD has been increasing at about $1.4 \mathrm{~ms}$ per century (Morrison \& Stephenson 1986). The actual value of the LOD departs from any long-term trend due to fluctuations on a time scale of decades. The epoch at which the mean solar day was exactly 86400 SI seconds was approximately 1820 . This is also the approximate mean epoch of the observations analyzed by Newcomb that resulted in the definition of the second of Ephemeris Time from which the SI second was derived (Newcomb 1895b). Fig. 4 shows more recent values of the excess length of day. 


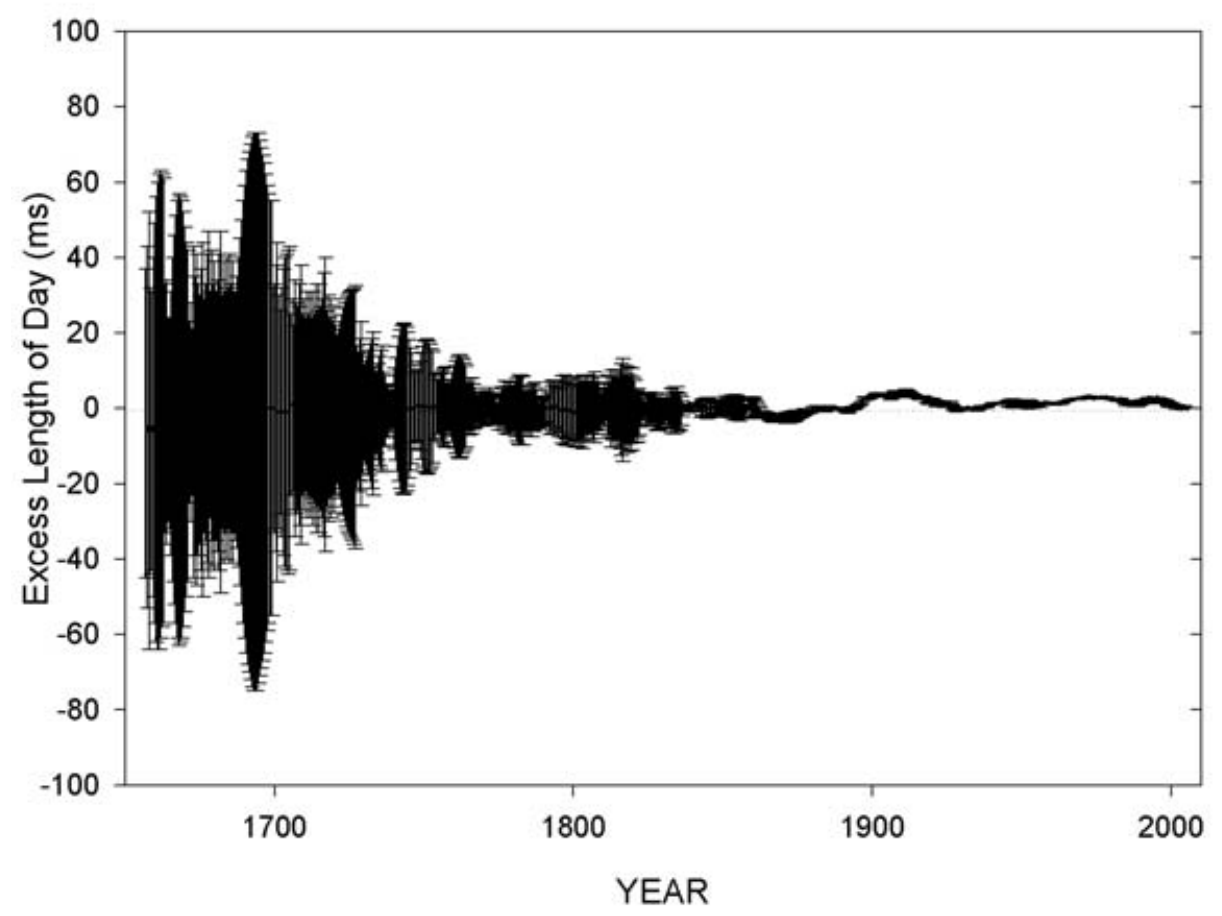

Figure 3. Variation of the excess length of day since 1620.

\subsection{Physical causes}

Three types of variation in the Earth's rotation have been identified: a steady deceleration, random fluctuations, and periodic changes. As early as 1695, Halley $(1693,1695)$ suspected an acceleration in the mean motion of the Moon from a study of ancient eclipses of the Sun. By the mid-eighteenth century, the lunar acceleration was fully established, and in 1754, Kant (1867) suggested that this acceleration might be an apparent phenomenon caused by a steady deceleration in the Earth's rotation due to tidal friction.

Jeffreys $(1920,1962)$ made the first quantitative estimate of global tidal friction in 1920. The rate of energy dissipation by tidal friction is now considered to correspond to a rate of increase in the length of day of $2.3 \mathrm{~ms}$ per century. To account for the observed deceleration, there must also be a component in the opposite direction of about $0.6 \mathrm{~ms}$ per century, which is possibly associated with changes in the Earth's shape caused by post-glacial rebound (Yoder et al. 1983) or with deep ocean dissipation (Egbert \& Ray 2000).

Evidence for a long-term deceleration in the Earth's rotation, extending over millions of years, also exists in coral fossils that exhibit both daily and annual growth rings (Wells 1963). The evidence suggests that the rate of deceleration was substantially the same then as it is now (Runcorn 1966). Besides a steady decrease, the Earth's rotation is subject to frequent small, apparently random changes. The irregular changes in speed may be correlated with physical processes occurring on or within the Earth. They include decade fluctuations with characteristic periods of $5-10$ years as well as variations, which occur at shorter time scales. 


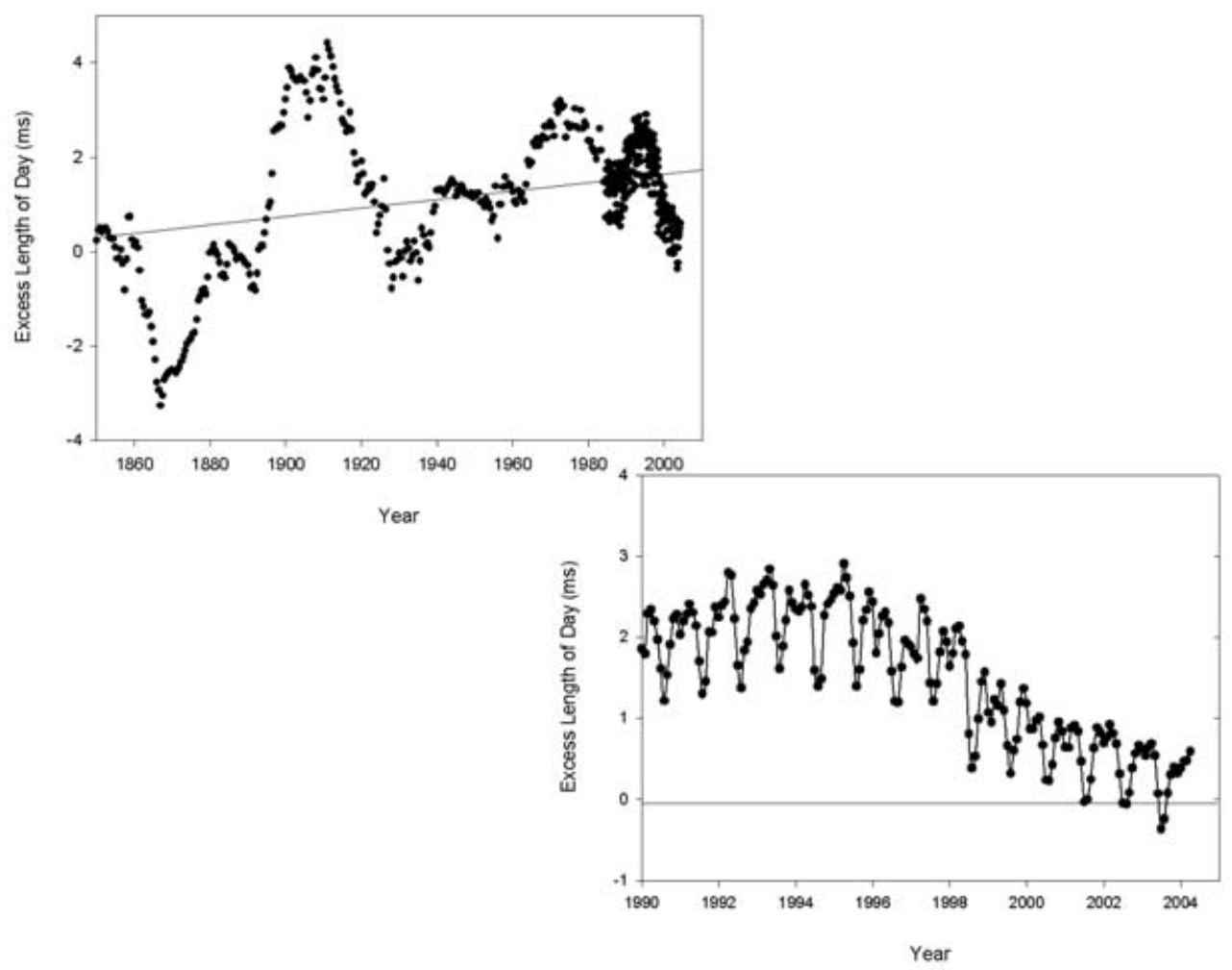

Figure 4. Variation of the excess length of day.

\section{Coordinated Universal Time}

The current definition of Coordinated Universal Time (UTC) was devised to satisfy the needs of two communities of users. Astronomers, geodesists and navigators would like a time connected with the angle of the Earth's rotation in space. Others, such as physicists and engineers, prefer a perfectly uniform time scale. Attempts to meet the needs of both communities led to the creation of UTC.

\subsection{History}

The term "Coordinated Universal Time" was introduced in the 1950s to designate a time scale in which adjustments to quartz crystal clocks were coordinated among participating laboratories in the USA and UK. The scale evolved over the years to the state where the BIH coordinated adjustments to an internationally accepted standard Coordinated Universal Time, designated UTC, that involved adjustments in both rate and epoch to stay in step with astronomical time.

The concept of the leap second, analogous to the leap day in the calendar, was proposed independently by Winkler (1968) and Essen (1968) at a meeting of the CIPM in 1968 (Commission Préparatoire pour la Coordination Internationale des Échelles de Temps 1968). It was proposed that integer steps of seconds replace the steps of $100 \mathrm{~ms}$ or $200 \mathrm{~ms}$ then being used. To meet the needs of navigators, it was suggested that coded information 
be incorporated in the radio time signals to indicate the difference between UTC and UT2.

The International Radio Consultative Committee (CCIR) formed an Interim Working Party to investigate requirements, submit proposals, and fix a date for the introduction of the new system. Specific proposals were made in Geneva in 1969, which were approved in January 1970. In its Recommendation 460 (International Radio Consultative Committee (CCIR) 1970a), the CCIR stated that (a) carrier frequencies and time intervals should be maintained constant and should correspond to the definition of the SI second; (b) step adjustments, when necessary, should be exactly $1 \mathrm{~s}$ to maintain approximate agreement with Universal Time (UT); and (c) standard signals should contain information on the difference between UTC and UT. The CCIR also decided to begin the new UTC system on 1 January 1972. At the IAU's 14th General Assembly in 1970, it was recommended that radio time signals should disseminate differences in the form of UT1 - UTC for navigational requirements.

Detailed instructions for the implementation of CCIR Recommendation 460 were drafted at a further meeting of Study Group 7 (International Radio Consultative Committee (CCIR) 1970b). The defining epoch of 1 January 1972, 0h 0m 0s UTC was set $10 \mathrm{~s}$ behind TAI, which was the approximate accumulated difference between TAI and UT1 since the inception of TAI in 1958, and a unique fraction of a second adjustment was applied so that UTC would differ from TAI by an integral number of seconds. The recommended maximum departure of UTC from UT1 was $0.7 \mathrm{~s}$, and the term "leap second" was introduced. The correction DUT1 was introduced, having integral multiples of $0.1 \mathrm{~s}$, to be embodied in the time signals such that, when added to UTC, they would yield a better approximation to UT1. In 1974, the CCIR increased the tolerance for UT1-UTC from $0.7 \mathrm{~s}$ to $0.9 \mathrm{~s}$.

The present UTC system is defined by ITU-R (formerly CCIR) Recommendation ITUR TF.460-5 (ITU - R Recommendations: Time Signals and Frequency Standards Emissions 1998): "UTC is the time scale maintained by the BIPM, with assistance from the IERS, which forms the basis of a coordinated dissemination of standard frequencies and time signals. It corresponds exactly in rate with TAI but differs from it by an integral number of seconds. The UTC scale is adjusted by the insertion or deletion of seconds (positive or negative leap seconds) to ensure approximate agreement with UT1." The interval between time signals of UTC is thus exactly equal to the SI second. A history of rate offsets and step adjustments in UTC is given at http://www.iers.org, and is shown in Fig. 5.

\subsection{Issues}

The primary reason for introducing the concept of the leap second was to meet the requirement of celestial navigation to keep the difference between astronomical time and atomic time small. However, this requirement has diminished because of the availability of electronic navigation systems, at the same time as the operational complexities of maintaining precise timekeeping systems without discontinuities have made the insertion of leap second adjustments increasingly difficult and costly. Recently created working groups of various international scientific organizations are now investigating the need to continue the leap second, with its technical inconveniences, or whether it would be better simply to discontinue the traditional relationship of timekeeping with the Earth's rotation. 


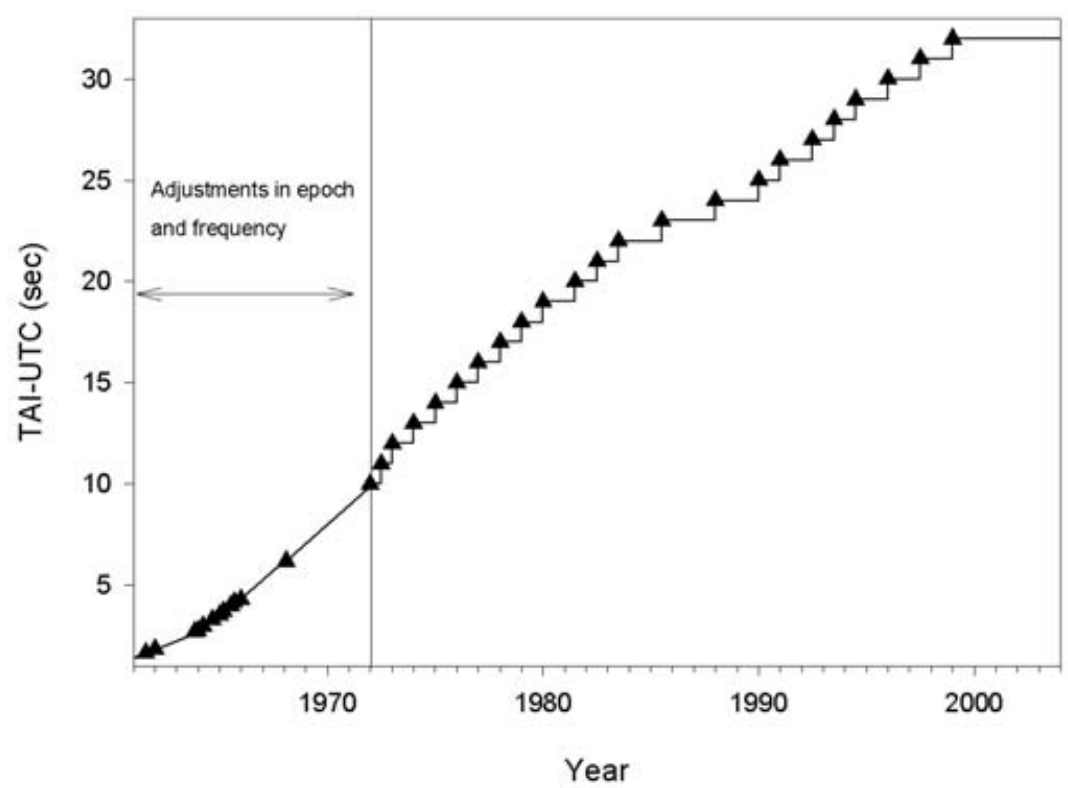

Figure 5. History of TAI-UTC showing the period of time when both rate and epoch were adjusted.

\subsubsection{Operational difficulties of preserving the leap second}

Modern commercial transport systems depend almost entirely on electronic navigation systems. It is likely that future systems will rely on this means of navigation even more, and it is clear that plans are currently being made to improve the satellite navigation systems to provide navigation accuracy, reliability, integrity and availability beyond current capabilities. Increasing worldwide reliance on satellite navigation for air transport is likely to demand systems free of unpredictable changes in timing epoch. Many telecommunications systems routinely rely on precise time synchronization. During the introduction of a leap second, communications can be lost until synchronization is reestablished. However, only systems that depend specifically on time are affected by the introduction of leap seconds; systems depending on frequency have little or no sensitivity to epoch. In high speed communications that time stamp messages at the sub-second level, one second can be a significant length of time. Clocks normally count from $59 \mathrm{~s}$ to 0 s of the next minute. Leap seconds require a sequence of $59 \mathrm{~s}, 60 \mathrm{~s}$, and then 0 s of the next minute. Many computer systems have a problem introducing the second labeled "60". A similar concern is that when dating events using the Julian Day (JD) or Modified Julian Day (MJD) including fractions of a day, a positive leap second would create a situation where two events 1s apart can receive identical dates when those dates are expressed with a numerical precision equivalent to $1 \mathrm{~s}$.

The possible introduction of one or two 61-s minutes per year into continuous site processes would directly affect synchronization if the leap seconds were not treated identically at the same instant at all cooperating sites. In some cases, the need to avoid disruptions has led to considerations of using nontraditional timekeeping systems, such as GPS Time or a time scale maintained by a private company. Continuing use of a nonuniform time scale including leap seconds in the face of these considerations could lead 
to the proliferation of independent uniform times adopted to be convenient for particular objectives.

\subsubsection{Operational difficulties of eliminating the leap second}

Some astronomers and satellite ground-station operators would prefer that leap seconds be retained. There is a significant amount of operational software at astronomical observatories and satellite ground stations that assumes implicitly that UT1 will always be within one second of the standard time. This assumption would no longer be true if leap seconds were eliminated. Fixing, testing and documenting this legacy code could be a major effort. It may also be difficult to change existing broadcast formats due to the prevalence of legacy hardware. Clocks exist that receive radio broadcast time signals to automatically display accurate time. These and similar devices might be affected adversely by a change in the broadcast format.

\section{Global Navigation Satellite System (GNSS) time scales}

Today, with GPS (Navstar GPS Space Segment/Navigation User Interfaces 2000) and GLONASS (GLONASS Interface Control Document, Ver. 4.0 1998), complemented by LORAN and other radio-navigation systems, celestial position determination is not as common as in the past. These systems and the augmentation systems they have fostered have been incorporated into various commercial applications. With extremely high accuracy and global coverage, satellite navigation systems have become a new public utility known by the general designation of Global Navigation Satellite Systems (GNSS).

\section{1. $G P S$}

The Global Positioning System (GPS), formally chartered in 1973, provides position with a precision on the order of a few meters or better and time with a precision on the order of 10 nanoseconds. Each satellite carries caesium or rubidium atomic clocks. The satellite and global tracking network atomic clocks are used to generate the time scale known as GPS Time, which is specified to be within one microsecond of UTC as maintained by USNO, modulo 1 second. The origin of GPS Time is midnight of 5/6 January 1980, so that TAI is ahead of GPS Time by $19 \mathrm{~s}$, a constant value. As of 1 January 2004, GPS Time is ahead of UTC by $13 \mathrm{~s}$. The algorithm defining the relationship between GPS Time and UTC includes a correction for leap seconds.

A new generation of GPS with enhanced capabilities, GPS III, is to be implemented in the future. The Earth-fixed orbit determination process for GPS, like virtually all other Earth-orbiting satellites, requires precise knowledge of UT1-UTC, and near realtime orbit determination must use predictions of this quantity. Today, these predictions are updated weekly (Bangert 1986). As GPS Time does not include leap seconds, the introduction of a leap second into UTC does not affect GPS users. The GPS operational control segment, however, must carefully account for the leap second step in UT1-UTC. Prior to a leap second event, two sets of Earth Orientation Parameters are provided to the GPS control segment. One is used up to the time a leap second is inserted and the second containing the new 1s step in UT1-UTC, is used after the leap second is inserted.

\subsection{GLONASS}

The Russian Global Navigation Satellite System (GLONASS) has many features in common with GPS (Daly 1991; Langley 1997). The system was fully deployed in early 1996 but currently there is not a full complement of operational satellites. The GLONASS design uses Moscow Time, (UTC $+3 \mathrm{~h})$ as its time reference instead of its own internal 
time. Thus, users of this system are directly affected by leap seconds. During the process of resetting the time to account for a leap second, the system is unavailable for navigation service because the clocks are not synchronized.

\subsection{GALILEO}

The Galileo satellite navigation system is under development by the European Space Agency. The start of full operation is planned for 2008. As the internal time scale for Galileo will be continuous without steps, it has been expected that the Galileo system time will be a realization of TAI. However, in a letter to the CCTF disseminated at its April, 2004 meeting (Hahn 2004), the director of the ESA Galileo Project Office expressed the hope that any decision to discontinue leap seconds should be made in the near future. The Galileo system could then adopt a realization of UTC as its internal reference time scale without facing any discontinuities.

\subsection{Future satellite systems}

The growing utilization of satellite systems and their internal time scales may gradually become the primary source of time for many practical applications. Options for the future integration of the civil time scale and the GNSS timescales may be examined in the context of several desirable objectives (Nelson et al. 2004). These are:

(a) standardization of time scales;

(b) internal time scales without steps (no leap seconds);

(c) reduction in the number of time scales;

(d) provision for access to standard civil time.

\section{Future time scale}

There exists a variety of options for the future of UTC. Some of these options are identified and discussed below.

\subsection{Maintain the status quo}

The advantage of maintaining the present form of UTC is that established timekeeping practices will not require modification. On the other hand, if leap seconds were continued, the required number and frequency can only increase. By 2100 there would be a need for nearly two leap seconds per year. The current emerging problems and the resulting dissatisfaction with leap seconds will only continue to grow. The operational impact and associated cost of maintaining leap seconds in complex timekeeping systems must be considered in evaluating their continued use in the future.

\subsection{Increase the tolerance between UT1 and UTC}

An increment of several leap seconds could be inserted into UTC every few years, a "leap minute" in about fifty years, or a leap hour hundreds of years from now. The advantage of this approach is that it would be relatively easy to adopt.

\subsection{Periodic insertion of leap seconds}

A time step could be inserted into UTC at a well-defined interval, such as on 29 February every four years. The advantage is that the date would be predictable. However, the number of leap seconds would not be predictable and large time steps would still be required. 


\subsection{Variable adjustments in frequency}

This alternative is similar to the original form of UTC that was abandoned. Introducing a variable atomic scale in step with solar time would cause significant disruptions to equipment and would not disseminate the unit of time, the SI second.

\subsection{Redefine the second}

This option would appear to be the most fundamental solution. However, it would be inconsistent with the usual practice in metrology, which is to adopt a new definition of a unit only when its realization under the old definition becomes the limiting source of experimental uncertainty and to maintain continuity between the old and new realizations. Changing the definition of the second to be closer to the current rotational second would alter the value of every physical measurement and render obsolete every instrument related to time. Moreover, the solution would be only temporary as the Earth continues to decelerate.

\subsection{Substitute TAI for UTC}

TAI is the fundamental atomic time scale from which other scales of uniform time are derived. TAI is related to UTC so that the increment to be applied to UTC to give TAI is equal to the total number of leap seconds plus $10 \mathrm{~s}$. The advantage of TAI is that it is a continuous, atomic time scale without steps. However, TAI is currently not easily available to the precise time user and, as TAI is currently ahead of UTC by an offset of $32 \mathrm{~s}$, a worldwide adjustment of clocks would be required if it were adopted as the scale of civil time. Promotion of two parallel time scales for civil timekeeping, one with leap seconds and one without would be potentially confusing. In addition, as UTC is recognized as the primary basis of civil time in resolutions of various international treaty and scientific organizations and by many conforming national legal codes, a worldwide change in the legal definition of time would be required if UTC were replaced by TAI.

\subsection{Discontinue leap seconds in UTC}

This option would permit continuity with the existing UTC time scale and would eliminate the need for future adjustments to complex timekeeping systems. If the current rate of deceleration of the Earth's rotation were to persist and no leap seconds were added, by 2050 the difference between UTC and UT1 would be about 1 min. By the end of the twenty-first century, the expected difference would be about $2.5 \mathrm{~min}$ (McCarthy \& Klepczynski 1999). However, these differences are minor compared with the difference between apparent solar time and mean solar time (up to $16.5 \mathrm{~min}$ ), mean solar time and clock time within a given time zone (nominally up to $30 \mathrm{~min}$ ), or Daylight Saving Time and Standard Time $(1 \mathrm{~h})$. It is thus unlikely that the growing difference between clock time and levels of daylight would be noticeable for the foreseeable future. Therefore, the elimination of leap seconds would have no practical effect on the correspondence between civil time and solar time or on contemporary social conventions. The use of UTC without leap seconds would retain all the advantages of TAI. The transition to a continuous UTC system might be planned for a future date sufficiently far in advance that changes to existing hardware and software, where necessary, could be accommodated within the normal maintenance and replacement schedules.

\section{Requirements of celestial navigation}

There remains the need to meet the requirements of celestial navigation. Possible options suggested in Nelson et al. (2001) for addressing this need if the current UTC 
system were revised are considered. Additional alternatives may be identified as the issue is debated.

The IERS/USNO provides daily and weekly predictions of UT1-UTC in Bulletin A, available on the Internet at http://www.iers.org. The estimated accuracies are $0.0017 \mathrm{~s}$ at 10 days and $0.0039 \mathrm{~s}$ at 30 days. In addition, long-term projections might be included in the nautical almanacs with less precision. With the usual yearly schedule of publication, the extrapolation should not bring errors exceeding $1 \mathrm{~s}$ (leading to a position error of $0.5 \mathrm{~km}$ at most). Through both short-term and long-term UT1 predictions, it would be possible to complement the information to navigators by disseminating a correction to the argument of the ephemerides, as is done currently with DUT1.

\section{Conclusions}

Modern timekeeping conventions have loosened the strict relationship between astronomical time based on the Earth's variable rotation and a uniform time scale enabling modern navigation and communication technology. It is likely that this tie will be loosened further in the future as the need for precise timekeeping increases and the practical requirements for a solar-based time decrease. It is also likely that the astronomical determination of the Earth's rotation angle will improve in the future, enabling improvements in the near real time orbit determination of satellite systems. There is no technical reason to continue the relationship between the two. It is reasonable to treat the rotation of the Earth as an angle measured in a system of uniform time.

A suitable compromise for the future could involve the following:

(a) Increase the tolerance between UT1 and UTC to one hour, enabling the predicted date for the adjustment by one hour to be made many years in advance.

(b) Acknowledge that UT1 is essentially a measure of the Earth's rotation angle to be determined astronomically, and improve the accessibility of this information to users by electronic means.

It is critical that the international community decide the future of UTC soon. The allocation of resources to implement future systems is being planned now, and critical decisions may involve the nature of future timekeeping systems.

\section{References}

Aoki S., Guinot B., Kaplan G. H., Kinoshita H., McCarthy D. D., Seidelmann P. K. 1982 Astron. Astrophys. 105, 359-361.

Bangert J. A. 1986 Proc. 4th International Geodetic Symposium on Satellite Positioning Austin, Tex.

Barnes J. A., Andrews D. H., Allan D. W. 1965 IEEE Trans. Instrum. Meas. IM-14, 228-232.

BIPM Com. Cons. Déf. Seconde 1970 5, 21-23; reprinted in Time and Frequency: Theory and Fundamentals, Natl. Bur. Stand. (U.S.) Monograph 1401974 (ed. B. E. Blair), U.S. Govt. Printing Office, Washington, D.C., 19-22.

Capitaine N., Guinot B., McCarthy D. D. 2000 Astron. Astrophys. 355, 398-405.

Capitaine N., Wallace P. T., McCarthy D. D. 2003 Astron. Astrophys. 406, 1135-1149.

Clemence G. M. 1948 Astron. J. 53, 169-179.

Commission Préparatoire pour la Coordination Internationale des Échelles de Temps 1968 Proc.-Verb. Com. Int. Poids et Mesures 36, Annexe 1, 109-113; reprinted in BIPM Com. Cons. Déf. Seconde 1970, 5, Annexe S 10, 121-125.

Daly P. 1991 Acta Astronautica 25, 399-406.

Egbert G. D., Ray R. D. 2000 Nature 405, 775-778.

Essen L. 1968 Metrologia 4, 161-165.

Essen L., Parry J. V. L. 1955 Nature 176, 280-282. 
Essen L., Parry J. V. L. 1957 Philos. Trans. R. Soc. London 250, 45-69.

Explanatory Supplement to the Astronomical Almanac rev. ed., (ed. P. K. Seidelmann), University Science Books, Mill Valley, Calif., 48.

Gerber E. A, Sykes R. A. 1966 Proc. IEEE 54, 103-116; reprinted in Time and Frequency: Theory and Fundamentals, Natl. Bur. Stand. (U. S.) Monograph 140 1974, (ed. B. E. Blair), U. S. Govt. Printing Office, Washington, D.C., 41-56.

GLONASS Interface Control Document, Ver. 4.01998 Coordination Scientific Information Center,Moscow.

Goldenberg H. M., Kleppner D., Ramsey N. F. 1960 Phys. Rev. Lett. 5, 361-362.

Guinot B. 1989 "Atomic Time," in Reference Frames for Astronomy and Geophysics (ed. J. Kovalevsky, I. I. Mueller \& B. Kolaczek), Kluwer, Boston.

Guinot B. 1994/1995 Metrologia 31, 431-440.

Guinot B. 2000 , in Polar Motion: Historical and Scientific Problems, IAU Colloquium 178 ASP Conference Series, Vol. 208 (ed. S. Dick, D. McCarthy \& B. Luzum), Astron. Soc. Pacific, San Francisco, 175-184.

Hahn J. 2004 European Space Agency Galileo Project Office, Letter to the CCTF (March 26, 2004).

Halley E. 1693 Philos. Trans. R. Soc. London 17, 913-921.

Halley E. 1695 Philos. Trans. R. Soc. London 19, 160-175.

IERS Conventions (1996) (ed. D. D. McCarthy), International Earth Rotation Service Tech. Note 21, Observatoire de Paris, Paris, 84.

International Radio Consultative Committee (CCIR) 1970a , XIIth Plenary Assembly CCIR International Telecommunication Union, Geneva, 227; reprinted in Time and Frequency: Theory and Fundamentals, Natl. Bur. Stand. (U. S.) Monograph 1401974 (ed. B. E. Blair), U. S. Govt. Printing Office, Washington, D.C., 31.

International Radio Consultative Committee (CCIR) 1970b , XIIth Plenary Assembly CCIR International Telecommunication Union, Geneva, 258a-258d; reprinted in Time and Frequency: Theory and Fundamentals, Natl. Bur. Stand. (U. S.) Monograph 1401974 (ed. B. E. Blair), U.S. Govt. Printing Office, Washington, D.C., 32-35.

ITU-R Recommendations: Time Signals and Frequency Standards Emissions 1998, International Telecommunication Union, Radio-communication Bureau, Geneva,15.

Jeffreys H. 1920 Philos. Trans. R. Soc. London A221, 239-264.

Jeffreys H. 1962 The Earth: Its Origin, History and Physical Constitution 4th ed., Cambridge University Press, New York, 514.

Kant I. 1867, in Sämmtliche Werke Leipzig, Vol. 1; Whether the Earth Has Undergone an Alteration of Its Axial Rotation, in Kant's Cosmogony 1968 (Translated by W. Hastie, (ed. W. Ley), Greenwood, New York, 157-165.

Lambeck K. 1980 The Earth's Variable Rotation Cambridge University Press, Cambridge.

Langley R. B. 1997 GPS World 8, 46-51.

Markowitz W., Hall R. G., Essen L., Parry J. V. L. 1958 Phys. Rev. Lett. 1, 105-107.

McCarthy D. D. 1991 Proc. IEEE 79, 915-920.

McCarthy D. D., Babcock A. K. 1986 Physics of the Earth and Planetary Interiors 44, 281-292.

McCarthy D. D., Klepczynski W. J. 1999 GPS World 10, 50-57.

Metrologia 1968 4, 43.

Metrologia 1971 7, 43.

Metrologia 1981 17, 70.

Morrison L. V., Stephenson F. R. 1986 , in Earth Rotation: Solved and Unsolved Problems (ed. A. Cazenave), Reidel, Boston, 69-78.

Natl. Bur. Stand. (U. S.) Tech. News Bull. 1949, 33, 17-24.

Navstar GPS Space Segment/Navigation User Interfaces, ICD-GPS-200C-004, 2000 ARINC Research Corporation, El Segundo, CA, 2000.

Nelson R. A., McCarthy D. D., Malys S., Levine S., Guinot B., Fliegel H. F., Beard R. L., Bartholomew T. R. 2001, Metrologia 38, 509-529.

Nelson R. A., McCarthy D. D., Gifford A., Bartholomew T. , submitted to 2004 Proceedings of the European Frequency and Time Forum

Newcomb S. 1895a Astronomical Papers Prepared for the Use of the American Ephemeris and 
Nautical Almanac Vol. VI, Part I: Tables of the Sun, U.S. Govt. Printing Office, Washington, D.C., 9.

Newcomb S. 1895b The Elements of the Four Inner Planets and the Fundamental Constants of Astronomy U.S. Govt. Printing Office, Washington, D.C., 1895, Chap. 2.

Runcorn S. K. 1966 Scientific American 215 26-33.

Scheibe A., Adelsberger U. 1936 Phys. Zeitschrift 37, 38.

Seidelmann P. K., Fukushima T. 1992 Astron. Astrophys. 265, 833-838.

Stephenson F. R. 1997 Historical Eclipses and Earths Rotation Cambridge University Press, 64. Stoyko N. 1937 C. R. Acad. Sci. 205, 79.

The International System of Units (SI), 7th ed. 1998 Bureau International des Poids et Mesures, Sèvres, 111-115.

Trans. Int. Astron. Union Vol. VIII 1954 Proc. 8th General Assembly, Rome, 1952 (ed. P. T. Oosterhoff), Cambridge University Press, New York, 66.

Trans. Int. Astron. Union Vol. XVI B 1977 Proc. 16th General Assembly, Grenoble, 1976 (ed. E. A. Muller \& A. Jappel), Reidel, Dordrecht, 60.

Trans. Int. Astron. Union Vol. XVII B 1980 Proc. 17th General Assembly, Montreal, 1979 (ed. P. A. Wayman), Reidel, Dordrecht, 71.

Trans. Int. Astron. Union Vol. XXI B 1992 Proc. 21st General Assembly, Buenos Aires, 1991 (ed. J. Bergeron), Reidel, Dordrecht, 41-52.

Trans. Int. Astron. Union Vol. XXIV B 2000 Proc. 24th General Assembly, Manchester, 2000, Astron. Soc. Pacific. San Francisco.

Wells J. W. 1963 Nature 197, 948-950.

Winkler G. M. R. 1968 Memorandum submitted to the ad hoc group meeting at the International Bureau of Weights and Measures (BIPM), 30 May 1968.

Yoder C. F., Williams J. G., Dickey J. O., Schutz B. E., Eanes R. J., Tapley B. D. 1983 Nature 303, $757-762$.

\section{Discussion}

Mikhail MARov: I am just curious: among other assumptions you just listed the communication problems. Is it still valid? Because we have, right now, so fast growing information technology and we need progress, so it seems that in reality wherever you are just at the moment, you could communicate easily through different kinds of channels.

Dennis MCCARThy: Most communication systems are based on interval and not epoch; those can always synchronise and they don't need to have a change in epoch from those that are right. There are some communication systems though that actually are based on time synchronisation in which the introduction of the second, if not done properly at the two sites causes confusion. We have instances of these things actually happening, where we'll have telephone cells drop out because somebody doesn't introduce the leap second the way they are supposed to, somebody forgets, or somebody does it and puts in the wrong sum, or changes their clock in the wrong direction - those are the problems. And then we have drop-outs which usually last only for a short time ... for 20 minutes while they adjust the clocks.

Myles Standish: You said right at the very end that we're getting away from the rotation of the Earth. When I try to explain what the different times are, I wish I could get rid of the word "time," and then for UT1 you'd use the rotation of the Earth, for UTC you'd use your wristwatch, etc., and I think there's something in that for me ...

Dennis MCCarthy: Well, I didn't have a chance to say it, but I think we need to get used to the fact that UT1 - UTC is just a way of expressing an angle, the Earth's rotation angle. It's no longer a time, UT1 is not really a time, it's a way to express the Earth's rotation angle and it should not be thought of as a real time scale. If we can get 
people to understand that, that's fine. So that we can neglect the Earth's rotation angle in our calculations, I suspect many people who write software and point telescopes are cheating by saying that UT1 is essentially equal to UTC. But in doing so they lose the accuracy of UT1. UT1 - UTC is known to an accuracy better than one second of time a tenth of a microsecond - it's really a disservice to keep that going.

Don KuRTZ: How much does the zero-point of the Earth's rotation angle change because of continental drift causing a change in the reference frame?

Dennis McCARThy: The two time systems have to be maintained. The definition of the terrestrial reference frame is designed so that there is no drift, so there's no risk. We compensate for it essentially by taking it out. At some level that affects the observations of UT1, but this is going to be a 5-, 10-, 15-year sort of thing, so it's pretty low.

Nicole Capitaine: Are you sure that now the Earth is accelerating? Are there some signs that this will change?

Dennis MCCARThy: As we speak the Earth is decelerating, but in the past it's been accelerating. We would look for it to change, we have had very substantial periods of acceleration in the past hundred years. They've always turned right around after a while and we get the question, "Is it ever going to unwind?" The answer is, "Yes, it probably will." It has in the past, it unwinds. It'll catch up, that problem is real.

Myles Standish: No negative leap seconds?

[laughter]

Dennis MCCARThy: No, we don't anticipate negative leap seconds. When we first thought this up we tried to tell people there would be leap seconds at the rate of two or three a year, and since that time we haven't had a leap second, so ...

[general laughter] 


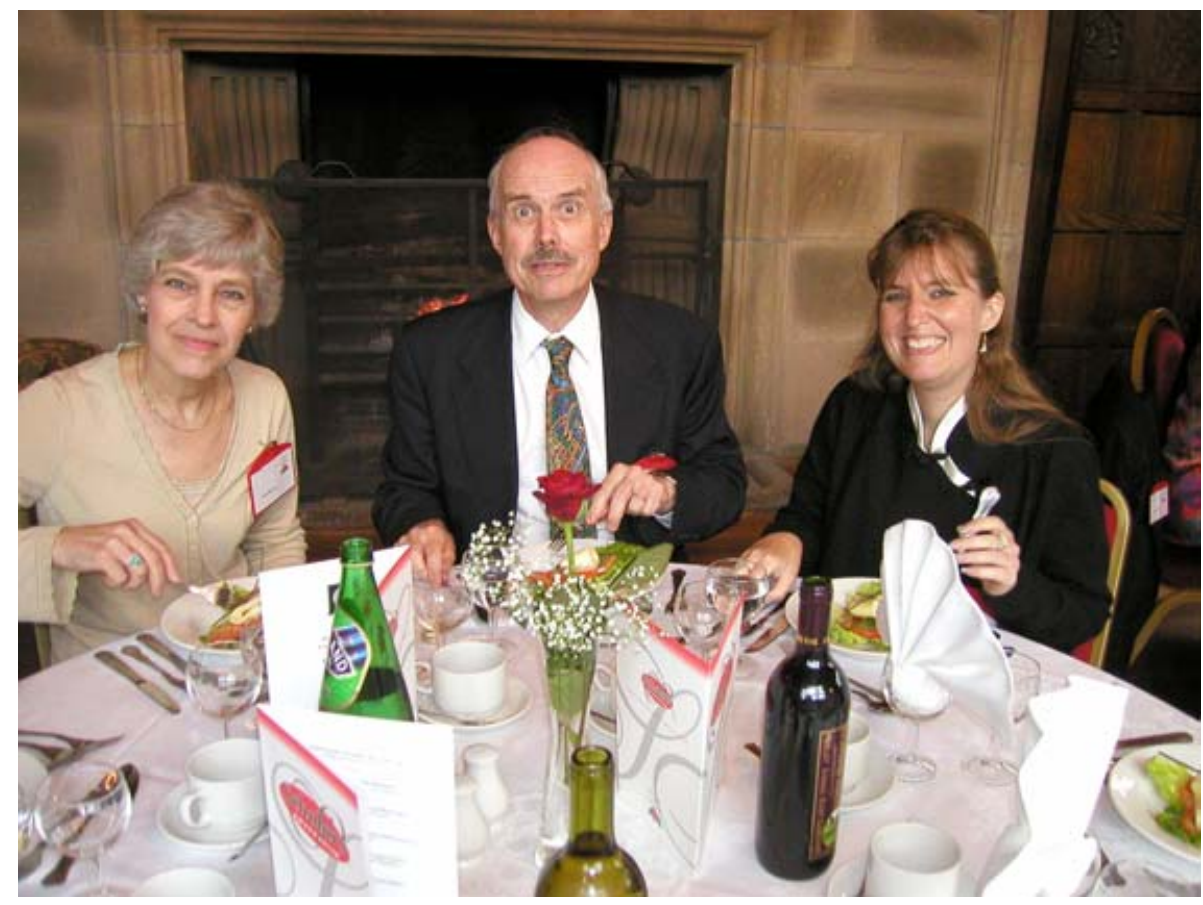

Diane, Dennis and Dierdre McCarthy

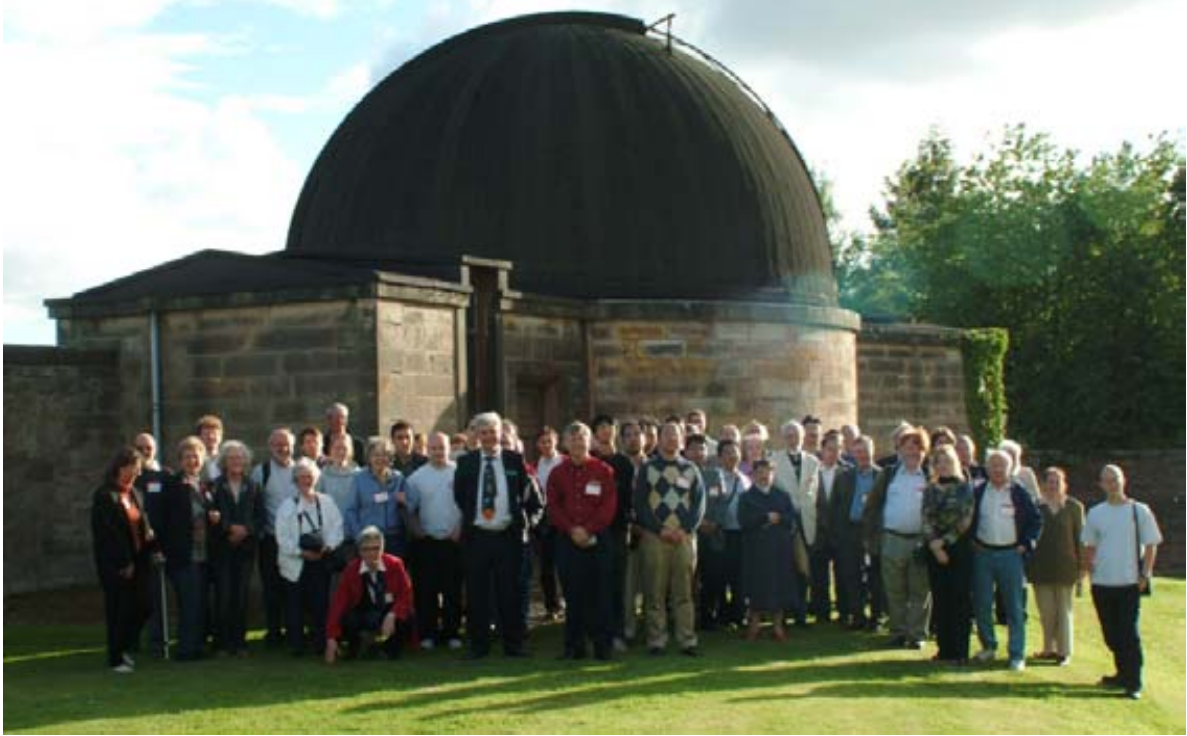

Conference tour of Stonyhurst College Observatory with Fintan O'Reilly 